\title{
The human conducting-system and its examination
}

\author{
R. E. B. HUDSON ${ }^{1}$ \\ From the Institute of Cardiology, University of London, and National Heart Hospital, London
}

It may be said that the survival of the race depends upon the reproductive organs but that the survival of the individual to three score and ten depends, as much as anything, upon the integrity of the conducting system of the heart, namely, the sinu-atrial pacemaker of Keith and Flack (1906 and 1907), and Lewis (1910-11), and the atrioventricular node with its bundle and branches, known as the His-Tawara system.

The writings of these giants of research have provided most of our present anatomical knowledge of this specialized neuromyocardial system which initiates and conducts the impulse for cardiac contraction. This knowledge has been available since the beginning of this century, but the advent of intracardiac surgery has brought the subject into the limelight once more for a new generation to study.

I can add nothing important to this knowledge; the object of this communication is to present a simple routine for examining the system which any pathologist can apply successfully when faced with the task. Hitherto, the matter has been somewhat shrouded in the obscurity created by the specialistas in so many other fields. It is no use pretending that the interpretation of histological appearances is easy but a good series of sections at least will allow an exchange of views. The results to be expected are exactly proportional to the care with which the examination is carried out. Hit-or-miss methods are

${ }^{1}$ Address: Pathology Department, National Heart Hospital, Westmoreland Street, London, W.1. a waste of time, and so in my view, are dissection techniques.

\section{SINU-ATRIAL NODE OF KEITH AND FLACK (PACEMAKER OF LEWIS)}

The pacemaker is a crescentic neuromyocardial structure pointed at both ends, which wraps around the anterior part of the arterial circle surrounding the junction of the superior vena cava with the right atrium. The thickest part of the node lies just below the highest point of this junction, which I have named the 'summit' of the right atrium; this landmark is easily identified in every heart (except perhaps in single atrium), and lies at the top of the crest ascending from the atrial appendage. Commonly, the widest part of the node lies to the right of this summit but it may lie more medially or laterally. To examine the node, the area enclosed by the dotted line in Fig. 1 is excised; it is therefore important not to open the superior vena cava (as is commonly done at necropsy) but to pack it lightly with cottonwool and fix in this position. In tiny hearts, the whole piece can be pinned out on a piece of cork, fixed, processed, detached and embedded in paraffin; close (not successive) serial sections are then cut from one vertical edge to the other. For adult hearts, it is sufficient to cut strips, as shown in the overhead view in Fig. 1, starting at the mouth of the superior vena cava. Each strip should be lettered separately and its origin recorded on a simple

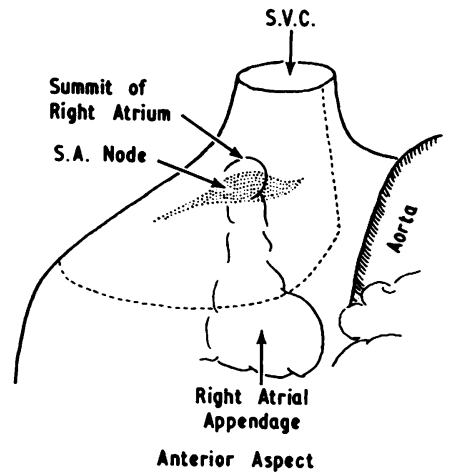

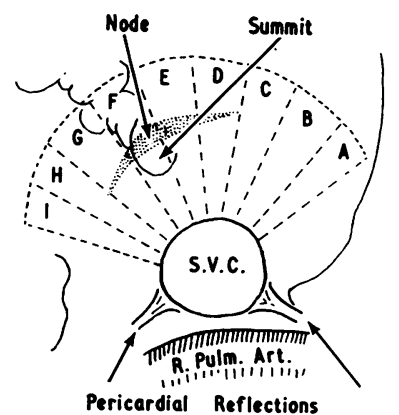

Overhead Viev

492
FIG. 1. Sketch to show the relation of the node to the 'summit' of the right atrium. The overhead view shows a convenient position in which to cut the strips $A$ to $I$. 

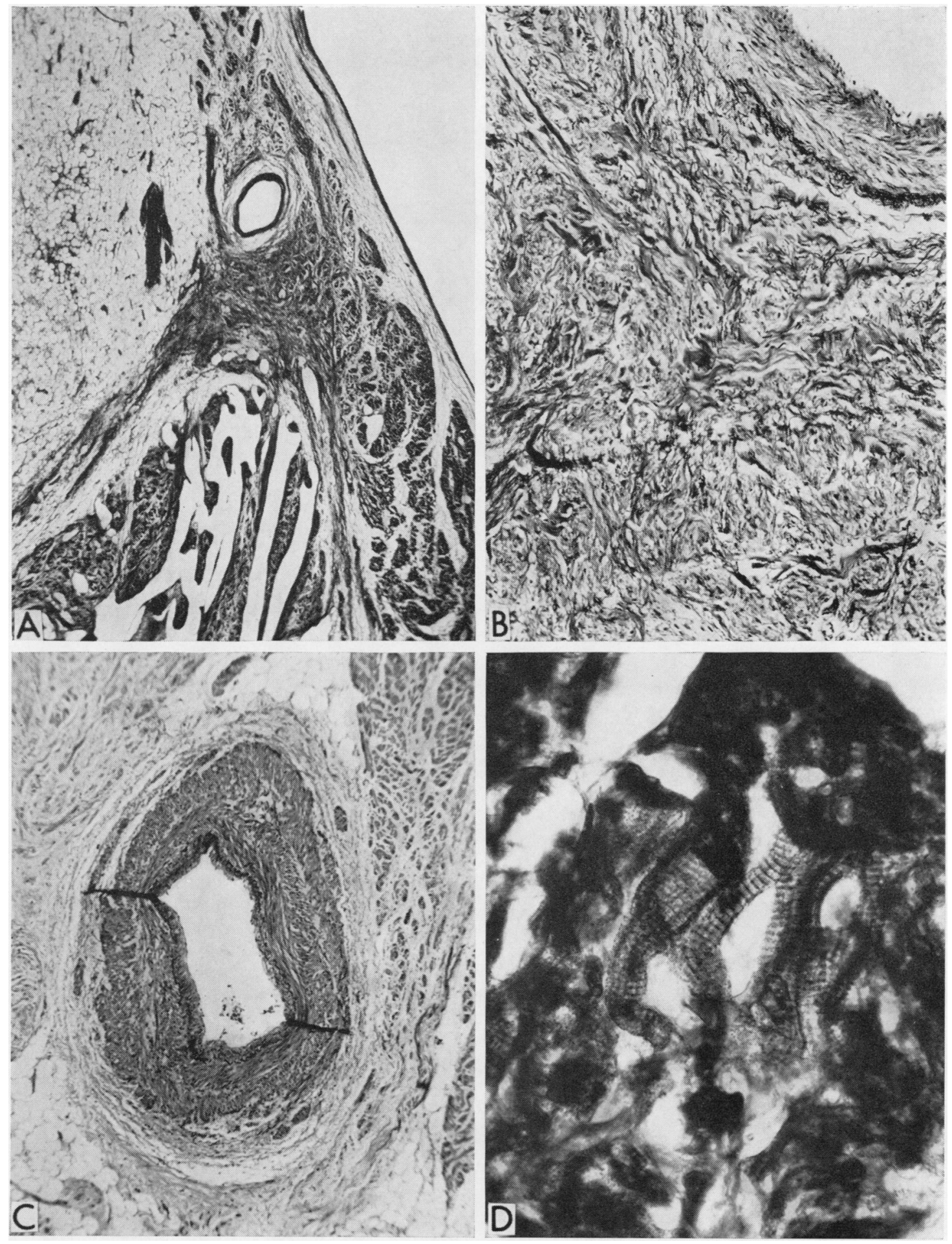

FIG. 2. General histological features of a sinu-atrial node.

A Main part of node with a large central artery, and extensions passing upwards to the superior vena cava and downwards to the atrium. Epicardium on left, lumen on right (Holmes' silver method $\times 15$ ).

B Detail of node structure. Artery at top right. Node muscle is paler than the wavy fibrous tissue (Verhoeff-van-Gieson $\times 100)$.

C Node artery showing three muscle layers in places (Verhoeff-van-Gieson $\times 40)$.

D Node muscle striations (Holmes' silver method $\times 900)$. 


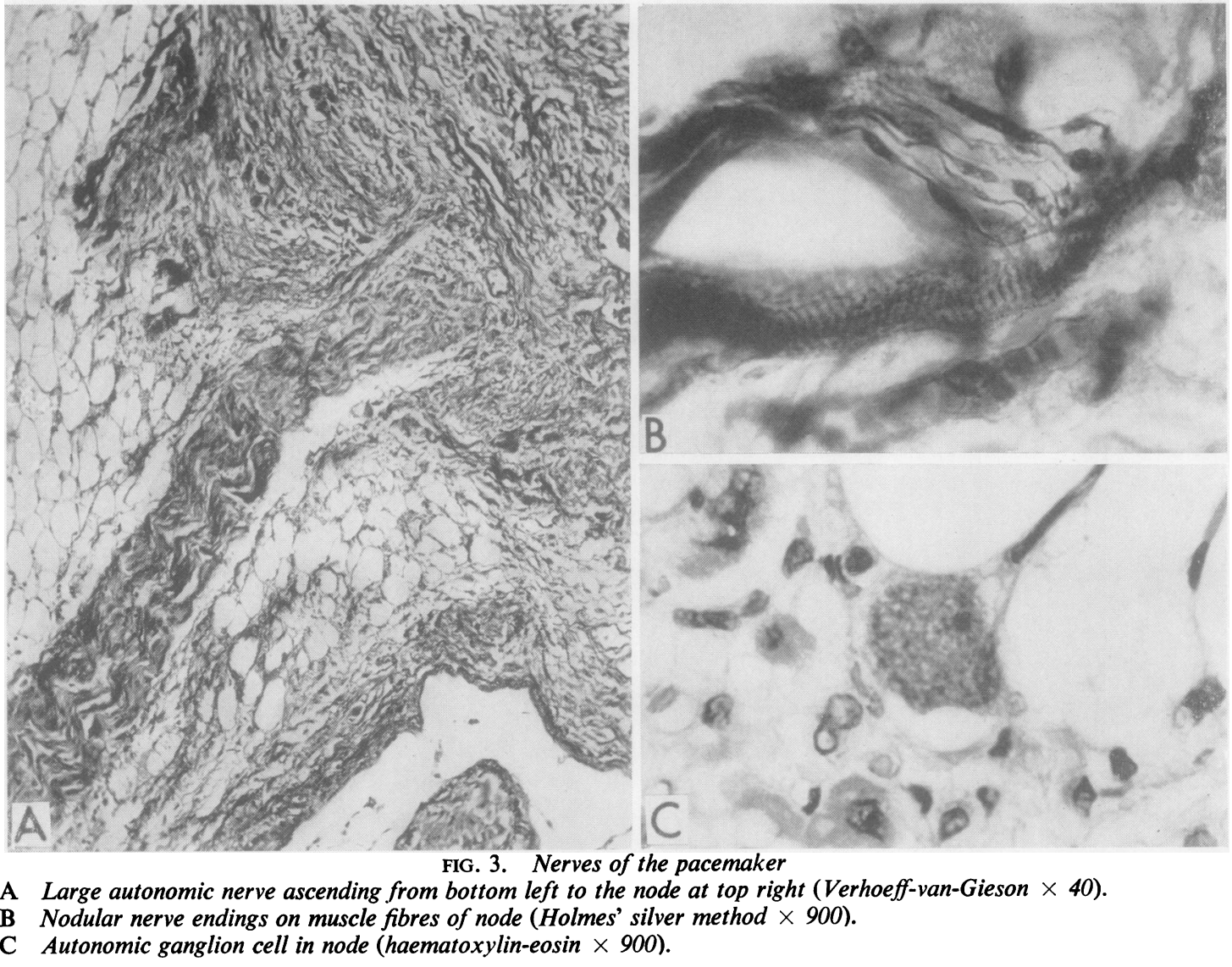

ground-plan. One or more sections from each strip will usually be enough. Staining the sections is a matter of choice; the writer has found the method of Verhoeff and van Gieson excellent.

Figure 2A shows the largest part of a node stained by Holmes' silver method. The artery is conspicuous and always a good guide to the node in sections; sometimes there are several arteries. Note the obvious extensions of the node downwards into the right atrium and upwards into the superior vena cava. Note also that the node is immediately beneath the epicardial fat (on the left); in this section the node is near the lumen too, with some intervening atrial myocardium. Figure 2B shows a portion of the node adjacent to the artery (top right); it shows a mixture of pale myocardial fibres, darker wavy fibrous tissue, and scattered elastic fibres. The muscle fibres tend to be smaller than those of the atrial myocardium, rather interwoven in the body of the node, and more vertically running at the periphery. Figure $2 \mathrm{D}$ is a silver preparation showing myocardial striations.

Nerves abound in the node, and autonomic ganglia lie in the adjacent epicardium. Figure 3A shows a large nerve bundle running from the lower left to enter the node at the top right of the picture. These nerves have their endings as fine nodular filaments ramifying on the surface of the muscle fibres (Fig. 3B); ganglion cells are not often seen in the node itself. Figure $3 \mathrm{C}$ shows an isolated example.

The blood supply, as already mentioned, comes from the arterial circle around which the node is disposed. This circle is fed by a branch from either the right or left circumflex coronary arteries (Fig. 4) and may be expected to suffer ischaemia if this is obstructed. The musculature of the artery in the node is often arranged in two or more layers; Fig. 2C shows a node artery with three layers in places.

\section{THE HIS-TAWARA SYSTEM}

The impulse from the pacemaker is thought to radiate into the right atrium to reach the atrioventricular node of Tawara, from which it travels along the bundle of His to be distributed to the ventricles by right and left branches.

Figure 5 is a diagram illustrating the general 


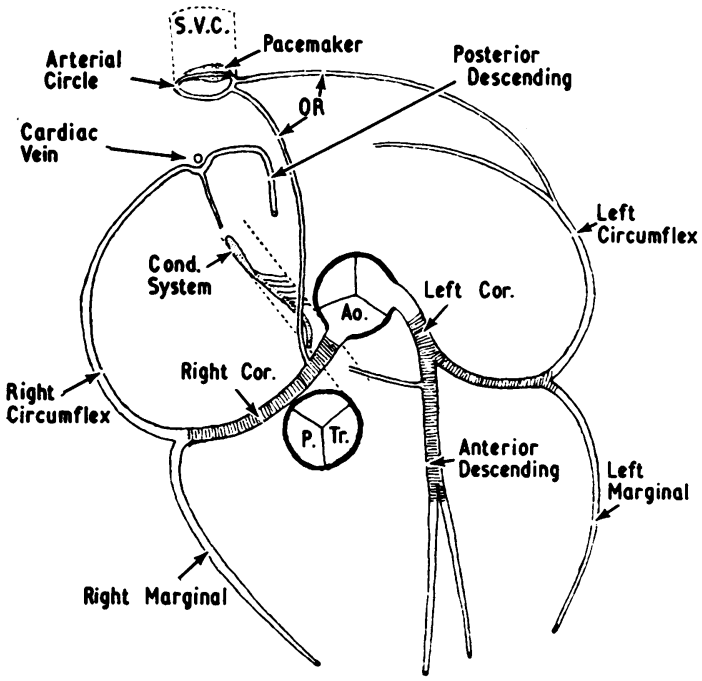

FIG. 4. Scheme of blood supply to the conducting system. The oblique parallel dotted lines represent the heart septa. The shaded areas of the arteries are where obstructions commonly occur.

anatomy of the system as seen from the right side of the heart septa. The atrioventricular node lies just beneath the endocardium of the right atrium between the entrance of the coronary sinus and the membranous portion (pars membranacea, membranous window) of the interventricular septum. The pars membranacea is divided by the attachment of the septal cusp of the tricuspid valve into two portions, atrial and ventricular. On the left side of the interventricular septum the membranous window fills in the area between the top of the muscular septum and the junction of the overhanging non-and right-coronary aortic valve cusps. The window therefore separates the left ventricle both from the right atrium and the right ventricle.

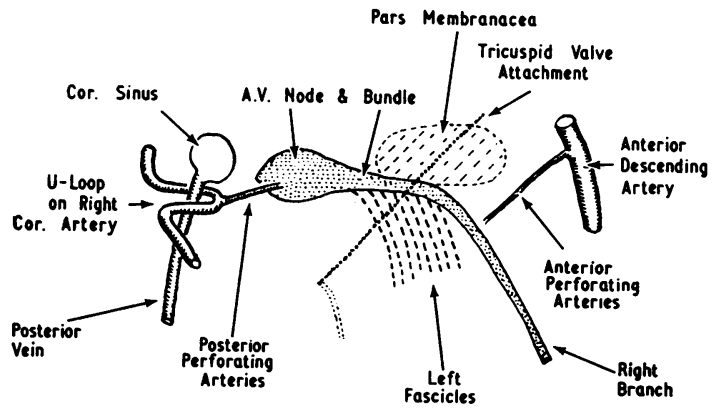

FIG. 5. Diagram showing the anatomical relations and blood supply of the His-Tawara system. The atrioventricular $(A . V$.$) node lies between the entry of the coronary$ sinus and the pars membranacea.
From the anterior end of the atrioventricular node emerges the bundle of His, which immediately penetrates the central fibrous body of the heart on its left side in order to reach the top of the muscular part of the interventricular septum where it lies as a compact cord running immediately below the posterior end of the membranous window. Soon after the bundle penetrates the fibrous body it

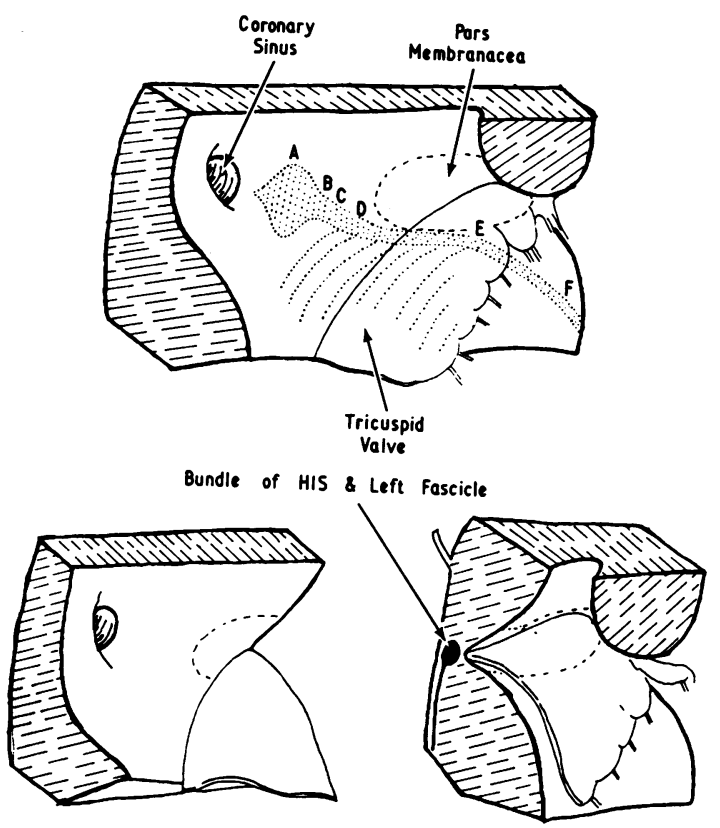

FIG. 6. Upper drawing shows the block of septum to be excised. The letters $A$ to $F$ indicate the origins of the sections shown in Figure 7.

Lower drawing shows how the vertical bisection passes through the centre of the system (in practice the two portions are left hinged at the bottom until the final embedding).

starts giving off a thin sheet of fascicles which pass to the left side of the septum, lying just deep to the plain muscle of the endocardium. When the left fascicles cease to come off the main bundle, the rest of the bundle moves over to the right side and proceeds as the right branch, descending as a compact cord and emerging towards the right ventricular subendocardium as it does so.

Figures 4 and 5 show how the blood supply to the atrioventricular node and posterior portion of the system comes from penetrating arteries derived from the U loop which the coronary artery (usually the right circumflex) sends into the back of the heart around the posterior cardiac vein as this vessel ascends to join the coronary sinus. The nourishment of the anterior part of the system comes from anterior 
penetrating arteries, usually derived from the left anterior descending coronary artery. No doubt the system also receives nutrition from the lumen blood, especially where it lies more superficially.

Figure 6 shows the technique of examination. First identify the membranous window by holding up the heart against a light shining into the opened left ventricle from the other side. Now excise the septum to include the coronary sinus opening and the membranous window as shown in the upper sketch. This block of tissue is then dehydrated slowly and thoroughly over the course of several days. At the final alcohol or first chloroform stage the block is trimmed and sketched from right and left sides for later reference. The whole left lower corner is then cut off to provide easy orientation of sections. Now cut the block vertically almost into two portions through the posterior part of the membranous septum; this cut will pass through the middle of the system. We have found it convenient to leave the two portions 'hinged' by a very thin layer of tissue at the bottom of this cut, although the lower sketch in Fig. 6 shows the two parts separated in order to display the system on the cut surface of the righthand piece. Dehydration and embedding of the 'hinged' block is completed, using vacuum to ensure penetration. When the block is removed from the last paraffin, the hinge is cut and both cut surfaces embedded downwards in fresh wax. This ensures that the first section from each block will show an easily identifiable part of the system, usually the main bundle and left fascicles. Sections are taken at intervals through each block; we have found that intervals of $0.5 \mathrm{~mm}$. are suitable for adult hearts, yielding about 60 to 70 sections, but closer serial section may be done according to facilities and enthusiasm. With very small hearts we prefer not to bisect the block but to section it whole at intervals of $0.25 \mathrm{~mm}$., starting at the anterior (membranous window) end. Staining is a matter of preference; Verhoff-van-Gieson stain is excellent but haematoxylin-eosin is quite good.

Figure 7 shows the appearances to be expected from a successful series of 60 sections $(3 \mathrm{~cm}$.) at points A, B, C, D, E, and F in Fig. 6 in an average heart. Note the cut-off lower corner marking the left ventricular side of the septum. The atrioventricular node (A) lies just under the right atrial myocardium, hugging the central fibrous body, which is black in the pictures. It is composed mainly of distinctive, rather thin, lacy-looking myocardial fibres and loose connective tissue in which are arteries, veins, lymphatics, and nerves. At its widest, the node is $5 \mathrm{~mm}$. or more high. From the node, one can usually trace fine superficial muscle fibres extending downwards to end near the tricuspid valve attach- ment, and also fibres entering the atrial myocardium above.

A few millimetres further forward the node becomes more compact and starts to penetrate the central fibrous body (B) to reach the ventricular $\frac{\bar{m}}{\bar{m}}$ septum. This is the area in which the system is $\overrightarrow{\mathbb{D}}$ interrupted in congenital heart block by excessive fibrous proliferation of the central fibrous body. \%

Having penetrated the fibrous body, the bundle $\overrightarrow{0}$ of His is now seen as a compact cord lying on the crest of the muscular interventricular septum in $\vec{\omega}$ the base of the pars membranacea (which is part of the central fibrous body); this is seen in C.

In this heart, as in most hearts examined by the $\vec{\sigma}$ writer, the bundle starts giving off left fascicles (D) quite early; these run down the left side of the endocardium just beneath the endocardial plain 0 muscle. At some distance from the origin, these fibres take on the appearance of Purkinje fibres, $\vec{z}$ large vacuolated-looking fibres with scanty peripheral fibrils, seen in the right-hand picture of $D$. Left fascicles continue to spring from the bundle and fan out over the left ventricle from the region of the membranous septum. Eventually, left fascicles cease coming off and just before this happens the $\mathrm{f}$ system appears to bifurcate (E); it will be clear from the foregoing that this is not true bifurcation, as featured in so many textbooks. During its course, the bundle sends branches directly into the top of the muscular septum also.

From this point forwards the main bundle, less its left fascicles, continues as the right branch, which moves over towards the right side of the muscular septum, eventually emerging in the subendocardial zone (F). Left fascicles will still be seen as they are sectioned running forwards from above but they appear lower in each succeeding section and $:$ eventually only the right branch will be seen. This is quite a distinct cord of myocardium, which can $\delta$ sometimes be picked out in a casual section.

Attention to Fig. 4 will show how coronary artery 웅 atheroma, occurring in the shaded areas most $D$ commonly, can compromise the blood supply to the whole conducting system.

The technique described is applicable to any heart, $\mathscr{N}$ whether normal or congenitally malformed. In $N$ general, the anatomy is the same; when a ventricular $\underset{\mathcal{E}}{N}$ septal defect is present, the bundle courses along 0 the very rim at the posterior end of the defect. $\ln _{\odot}$ this position it is highly vulnerable to trauma in $\frac{\vec{D}}{\mathrm{D}}$ the surgical repair of ventricular or ostium primum $\stackrel{?}{+}$ defects. Figure 8 (A and B) shows the right-and left- 0 sided views of a heart in which a large ostium primum atrial septal defect has been patched over. $\stackrel{\square}{\circ}$ Note the sutured cleft in the mitral valve in B; this $\mathbb{\mathbb { D }}$ cleft is the rule in this type of defect (as it is in 

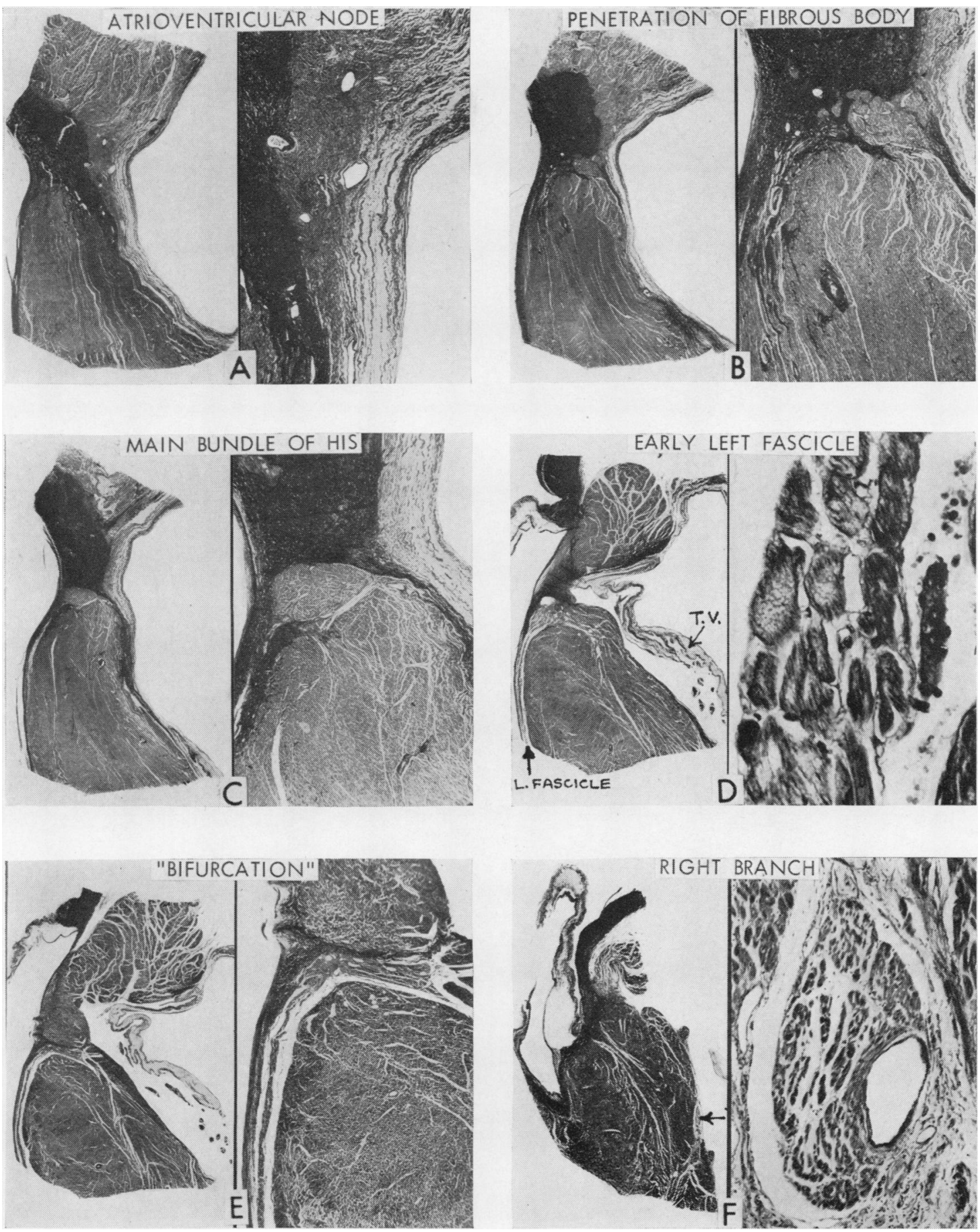

FIG. 7. Histological appearances of an average His-Tawara system. The letters A to F correspond with those on Fig. 6;

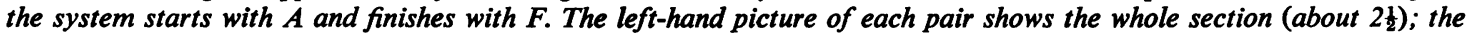
right-hand picture illustrates more detail (Verhoeff-van-Gieson). 

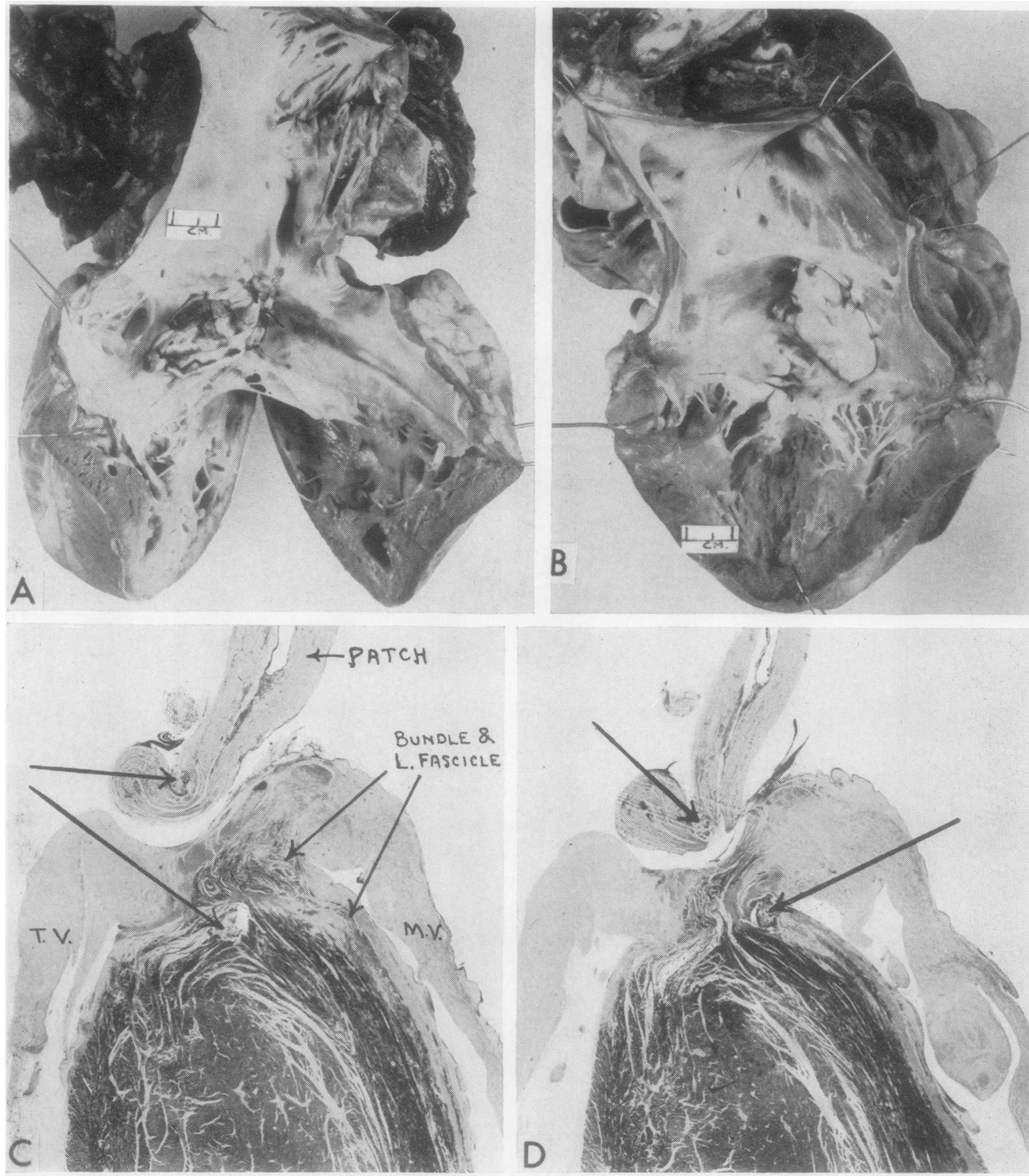

FIG. 8. Surgical trauma to His-Tawara system.

$\mathrm{A}$ and $\mathrm{B}$ show the right and left-side views of an ostium primum atrial septal defect. In $\mathrm{C}$ and $\mathrm{D}$ the unlettered arrows indicate sutures (haematoxylin-eosin $\times 9$ ).

single atrium). In $A$, the lower sutures near the coronary sinus opening are in the danger area. The arrows in $C$ show a stitch just below the main bundle of His as it gives off an early left fascicle and another suture in the patch. In $D$, the lower suture is in the path of the left fascicle. Other sections showed extensive haemorrhagic damage to the left $\stackrel{\oplus}{+}$ fascicles.

REFERENCES

Keith, A., and Flack, M. W. (1906). Lancet, 2, 359. - (1907). J. Anat. Physiol., 41, 172

Lewis, T. (1910-11). Heart, 2, 23. 